\title{
Effects of Cinnamon on Anthropometry Status and Headache Disability of Migraine Patients: A Randomized Double-blind Placebo-controlled Trial
}

\author{
Azadeh Zareie \\ Isfahan University of Medical Sciences \\ Mohammad Bagherniya \\ Isfahan University of Medical Sciences \\ Manoj Sharma \\ University of Nevada Las Vegas \\ Fariborz Khorvash \\ Isfahan University of Medical Sciences \\ Akbar Hasanzadeh \\ Isfahan University of Medical Sciences \\ Gholamreza Askari ( $\square$ askari@mui.ac.ir) \\ Isfahan university of sciences https://orcid.org/0000-0003-1194-9687
}

Research article

Keywords: Cinnamon, Migraine, Anthropometry, Migraine Disability Assessment

Posted Date: July 6th, 2020

DOI: https://doi.org/10.21203/rs.3.rs-39330/v1

License: (1) This work is licensed under a Creative Commons Attribution 4.0 International License. Read Full License 


\section{Abstract}

Background: Migraine is a common type of primary headache that is highly disabling and is possibly associated with obesity. Increasing body mass index (BMI) seems to be a risk factor for migraine attacks. Cinnamon has anti-inflammatory, neuroprotective, and anti-obesity effects. Thus, this study aimed to assess the effects of cinnamon on anthropometry status and headache disability of migraine patients.

Methods: Fifty patients with migraine were randomized to receive either cinnamon powder, three capsules/day each containing $600 \mathrm{mg}$ of cinnamon or three placebo capsules/day each containing $100 \mathrm{mg}$ of corn starch (control group) for two months. Height, body weight (BW), waist circumference (WC), and hip circumference (HC) were measured at baseline and the end of the study. Furthermore, Minimal or Infrequent Disability (MIDAS) and Headache Daily Result (HDR) Questionnaire were recorded.

Results: After follow-up, BW and BMI did not change in the intervention group, however, both of them significantly increased in the placebo group. The differences between the two groups were statistically significant $(p=0.001)$. WC significantly decreased in the cinnamon group and remarkably increased in the control group; the difference between groups was significant $(p<0.001)$. Furthermore, $\mathrm{HC}$ and WHR significantly decreased in the intervention group than the placebo group $(\mathrm{p}=0.001)$. HDR and the total score of disability in migraine patients were significantly decreased in the intervention versus the control group $(p<0.001)$.

Conclusion: Cinnamon seems to have beneficial effects on BW, BMI, WC, and $\mathrm{HC}$ and it reduces the headache disability of migraine patients. More randomized controlled trials should be undertaken to confirm these effects.

\section{Introduction}

Migraine is one of the most common primary headaches. It is known as the third-highest disabling disorder worldwide [1]. It is prevalent in approximately $12 \%$ of the Western world's population and $14 \%$ of the Iranian population [2]. On the other hand, overweight and obesity are current and increasing health problems with more than 1.9 billion overweight and obese adults in over the world [3]. Obesity is associated with an increased risk of several chronic diseases such as diabetes, dyslipidemia, hypertension, cardiovascular diseases, cancer, and pain disorders like headaches [4].

A growing body of evidence has indicated that overweight and obesity may be associated with a higher risk of migraine. Higher body mass index (BMI) is linked to more severe headaches, their increased frequency, and poorer quality of life among migraine patients $[5,6]$. Since migraine prevention medications can increase the body weight (BW) and BMI [7], and increased BW and BMI have adverse effects on migraine symptoms, finding a safe, novel and practical strategy to control weight gain to prevent the progression of headaches in patients is necessary. Despite the different weight control strategies, complementary and alternative approaches with comparatively fewer side effects have attracted significant attention. Hence, herbal supplementation might be used as easier and more practical alternative approach [8].

The bark of various cinnamon species as the most important and popular spice has been used worldwide for cooking and in traditional and modern medicines. Several potential health benefits of cinnamon have been known including anti-inflammatory, antioxidant, anti-neuroinflammatory, neuroprotective, insulin-sensitizing, and anti-obesity [9]. Cinnamaldehyde, polyphenols, and flavanols are the most important constituents of cinnamon that they have antioxidant and anti-obesity properties [8].

Previous studies indicate that cinnamon has protective effects against metabolic syndrome's aspects, non-alcoholic fatty liver disease (NAFLD) and obesity [10-12]. Therefore, it seems that BW and BMI control with cinnamon consumption can be a preventive approach for migraines. To the authors' knowledge, there has been no prior randomized controlled trial to assess the effects of cinnamon supplementation on changing anthropometry indices and disability in migraine patients. Hence, the main aim of the present study was to evaluate the effect of cinnamon supplementation on anthropometry status and headache disability of migraine patients.

\section{Materials And Methods}

\subsection{Sample size}


Sample size of this randomized double-blinded placebo-controlled trial was based on $80 \%$ power, an alpha level of 0.05 , and a potential dropout rate of $10 \%$. It was calculated that 50 participants (i.e., 25 subjects in each group) would be needed to detect $20 \%$ differences between-group [13].

\subsection{Participants}

A total of 50 eligible participants who visited one expert neurologist at Khorshid and Imam Mousa Sadr Clinics, Isfahan University of Medical Sciences, Isfahan, Iran were recruited. The migraine condition was confirmed by a single expert neurologist based on the third edition of the International Classification of Headache Disorders (ICHD-3) [14].

Inclusion criteria were as follows: 1) aged 20-50 years old; 2) migraine without aura that was diagnosed by one expert neurologist; 3) willingness to participate in the study after voluntary informed consent; 4) having normal to moderate anxiety, stress, and depression status according to the DASS-21 questionnaire [15].

Patients were excluded if they had one these criteria: 1 ) patients with tension-type headache; 2) migraine with aura; 3) chronic diseases such as chronic kidney diseases and gastrointestinal diseases; 4) patients with a history of cinnamon sensitivity and allergy; 5) taking any antioxidants, cinnamon supplement and anti-inflammatory drugs; 6) menopause, pregnant or breastfeeding; 7) not taking any weight loss medication and not having a weight loss diet; and 8) who did not provide voluntary informed consent.

\subsection{Study design and Intervention}

This study was designed as a double-blinded placebo-controlled randomized trial. All participants were randomized into intervention and control groups. Permuted four-block randomization method according to gender in a 1:1 ratio applying the table of random numbers was used for randomization. A trained nutritionist carried out enrolling and allocating subjects to groups. Investigators and participants were blinded to the randomization codes until the completion of final analyses.

Patients in each group received three cinnamon or placebo capsules after each main meal each day for 60 days. The cinnamon capsules contained $600 \mathrm{mg}$ of dried Cinnamomumzeylanicum bark powder (Ceylon cinnamon) $+100 \mathrm{mg}$ of corn starch and placebo capsules contained $100 \mathrm{mg}$ of corn starch, these capsules were produced with similar shape, color and odor by Faculty of Pharmacy, Isfahan University of Medical Sciences.

All patients were instructed to take their usual headache treatment medications because the adjuvant treatment alone is not ethical to adminsiter. They were also requested to refrain from taking nonsteroidal anti-inflammatory drugs (NSAIDs) and not change their medication type and dose unless prescribed by their neurologist. In addition, patients were encouraged to maintain their usual diet and routine physical activity throughout the intervention period.

\subsection{Socio-demographic and dietary intakes}

Demographic information was collected by a well-trained nutritionist. Using face-to-face interviews and a standard questionnaire, demographic data including age, sex, economic status, history of medications, family history of migraine and baseline anthropometry status were taken from all participants. Also, 3-day dietary records (2-week days and 1-week end) at the beginning and the end of the trial were completed by all participants. Dietary intakes were entered into the Nutritionist IV software that was modified for Iranian foods.

\subsection{Headache daily result and disability assessment}

Headache daily result (HDR), the mean duration of migraine attacks per day [5], and the disability of migraine were determined by an experienced neurologist. Migraine disability was evaluated through a short and self-administered questionnaire thatquantified headache-related disability (MIDAS questionnaire). This questionnaire had 5 questions; the total score is calculated based on the number of days marked against each question [16]. The reliability and validity of the MIDAS questionnaire has been established in Iranian patients [17]. Based on the total score, four grades were considered: Grade I with $0-5$ score that meant little or no disability; Grade II with 6-10 score that meant mild disability; Grade III with 11-20 score that meant moderate disability; and Grade IV with more than 21 score that meant severe disability [16]. 


\subsection{Anthropometric assessment}

Bodyweight (BW) in kilograms (kg) was measured by a calibrated digital scale with a measurement precision of $0.1 \mathrm{~kg}$ while wearing lightweight clothing and no shoes. The height $(\mathrm{cm})$ was measured by a non-elastic tape and a measurement precision of $0.1 \mathrm{~cm}$. BMl as the ratio between weight in kilograms and height in meters squared $\left(\mathrm{kg} / \mathrm{m}^{2}\right)$ was calculated. Waist circumference (WC) and hip circumference (HC), as a measure of aggregate fat, were assessed by a non-flexible tape to the nearest of $0.1 \mathrm{~cm}$. WC was quantified in a standing position at the midpoint between the highest point of the iliac crest and lower part of the costal margin at the mid-axillary line. HC was evaluated from where the buttocks protrude the most. Waist-to-hip ratio (WHR) was calculated from the division of waist circumference in hip circumferences.

\subsection{Statistical analyses}

The statistical analyses of data were carried out using the Statistical Package for the Social Sciences (SPSS) (Windows version 22.0, IBM Corp., Armonk, NY, USA). At first, data normality was determined by the Kolmogorov-Smirnov distribution test. Then, qualitative variables were compared by the chi-square test, which were expressed as frequencies (percentages). To compare mean differences between two groupsat baseline, independent-sample t-test or Mann-Whitney test were performed. The pairedsamples t-test or Wilcoxon test were used to distinguish the effect of the intervention between the two groups. Analysis of covariance (ANCOVA) was carried out to distinguish the effect of the intervention between the two groups, adjusted for age and sex. Data were reported as mean \pm SE. The test level for the statistical significance of differences in groups of the study was defined as $p \leq 0.05$.

\section{Results}

From 114 patients who were diagnosed by one expert neurologist in the headache clinic, 50 subjects met the eligibility criteria. During the study period (60 days), in the cinnamon group two participants were excluded because of allergic reaction including itching, and two participants in the cinnamon group and three participants in the placebo group refused to continue the study No other side effects including constipation, diarrhea, nausea, and vomiting were seen except allergiv reaction in two participants. Finally, $86 \%$ of the participants ( 21 in the intervention group and 22 in the control group) completed the study and have been included in the final analysis (Fig. 1).

\subsection{Participant characteristics and nutrient assessment}

At baseline, no significant differences were observed between two groups regarding anthropometric variables, age, gender, economic status, family history of migraine, and medications used (Table 1). According to the current findings, dietary approaches, the glycemic index of the diet, and the balance between the intake of essential fatty acids could be considered as effective strategies in headache/ migraine [18]. Therefore, in the 3-day dietary records, we considered total energy, macronutrients, and some micronutrients intake that they are effective in losing weight. After data analysis, no significant differences between groups were found in terms of dietary macro and micronutrients (Table 2). 
Table 1

Baseline demographic characteristics of migraine patients in groups of the study

\begin{tabular}{|c|c|c|c|}
\hline Variable & Cinnamon group $(n=21)$ & Placebo $(n=22)$ & $P$ Value \\
\hline Age $($ mean \pm SE $(y))$ & $37.13 \pm 1.66$ & $39.36 \pm 1.46$ & $0.32^{\mathrm{a}}$ \\
\hline \multicolumn{4}{|l|}{ Gender (number (\%)) } \\
\hline Female & $16(76.19)$ & 19 (86.36) & \multirow{2}{*}{$0.35^{\mathrm{b}}$} \\
\hline Male & $5(23.80)$ & $3(13.63)$ & \\
\hline Family history of migraine (number (\%)) & $18(85.71)$ & $15(68.18)$ & $0.15^{\mathrm{b}}$ \\
\hline Weight (mean \pm SE $(\mathrm{kg})$ ) & $70.02 \pm 2.42$ & $70.50 \pm 3.46$ & $0.64^{a}$ \\
\hline Height (mean \pm SE $(\mathrm{cm})$ ) & $164.13 \pm 2.27$ & $161.45 \pm 1.60$ & $0.34^{\mathrm{a}}$ \\
\hline WC $($ mean \pm SE $(\mathrm{cm}))$ & $88.79 \pm 1.96$ & $93.00 \pm 2.80$ & $0.22^{\mathrm{a}}$ \\
\hline $\mathrm{HC}($ mean $\pm \mathrm{SE}(\mathrm{cm}))$ & $105.47 \pm 1.33$ & $101.06 \pm 4.63$ & $0.36^{a}$ \\
\hline \multicolumn{4}{|l|}{ Economic status (number (\%)) } \\
\hline Very low income & $5(23.80)$ & $1(4.54)$ & $0.72^{\mathrm{C}}$ \\
\hline Low income & 7(33.33) & $9(40.90)$ & \\
\hline Average income & $8(38.09)$ & $12(54.54)$ & \\
\hline High income & $1(4.76)$ & 0 & \\
\hline \multicolumn{4}{|l|}{ Medications (number (\%)) } \\
\hline \multicolumn{4}{|l|}{ Antidepressants } \\
\hline Tricyclic Antidepressant & $2(9.52)$ & $4(18.18)$ & $0.31^{b}$ \\
\hline SSRI & $3(13.63)$ & $3(13.63)$ & $1^{b}$ \\
\hline SNRI & $2(9.52)$ & $2(9.09)$ & $1^{b}$ \\
\hline Antiepileptic & 7 (33.33) & $10(45.45)$ & $0.32^{b}$ \\
\hline Gabapentin & $2(9.52)$ & $3(13.63)$ & $0.50^{\mathrm{b}}$ \\
\hline Beta-blockers & $5(23.80)$ & $8(36.36)$ & $0.32^{b}$ \\
\hline
\end{tabular}


Table 2

Dietary intake and physical activity of participants

\begin{tabular}{|c|c|c|c|c|c|c|c|c|c|}
\hline \multirow[t]{2}{*}{ Variable } & \multicolumn{4}{|c|}{ Cinnamon group $(n=21)$} & \multicolumn{4}{|c|}{ Placebo $(n=22)$} & \multirow{2}{*}{$\begin{array}{l}P \\
\text { Value }^{\mathrm{b}}\end{array}$} \\
\hline & Baseline & $\begin{array}{l}\text { After } \\
\text { intervention }\end{array}$ & $\begin{array}{l}\text { Mean } \\
\text { Difference }\end{array}$ & $\begin{array}{l}\text { P- } \\
\text { Value }^{a}\end{array}$ & Baseline & $\begin{array}{l}\text { After } \\
\text { intervention }\end{array}$ & $\begin{array}{l}\text { Mean } \\
\text { Difference }\end{array}$ & $\begin{array}{l}\text { P- } \\
\text { Value }^{a}\end{array}$ & \\
\hline Energy (Kcal) & $\begin{array}{l}1854 \pm \\
168.27\end{array}$ & $\begin{array}{l}1896 \pm \\
129.97\end{array}$ & $\begin{array}{l}42.53 \pm \\
130.92\end{array}$ & 0.74 & $\begin{array}{l}1919.71 \\
\pm 96.43\end{array}$ & $\begin{array}{l}1967.88 \pm \\
78.01\end{array}$ & $\begin{array}{l}48.17 \pm \\
102.53\end{array}$ & 0.64 & 0.97 \\
\hline $\begin{array}{l}\text { Carbohydrate } \\
\text { (gr) }\end{array}$ & $\begin{array}{l}287.53 \\
\pm 37.69\end{array}$ & $\begin{array}{l}281.05 \pm \\
38.74\end{array}$ & $\begin{array}{l}-6.48 \pm \\
11.24\end{array}$ & 0.57 & $\begin{array}{l}272.01 \\
\pm 14.06\end{array}$ & $\begin{array}{l}262.10 \pm \\
10.08\end{array}$ & $\begin{array}{l}-9.90 \pm \\
13.28\end{array}$ & 0.46 & 0.85 \\
\hline Glucose (gr) & $\begin{array}{l}6.68 \pm \\
0.91\end{array}$ & $7.36 \pm 1.21$ & $\begin{array}{l}0.67 \pm \\
1.55\end{array}$ & 0.66 & $\begin{array}{l}8.44 \pm \\
0.89\end{array}$ & $6.98 \pm 0.78$ & $\begin{array}{l}-1.46 \pm \\
1.01\end{array}$ & 0.16 & 0.25 \\
\hline Fructose (gr) & $\begin{array}{l}7.60 \pm \\
1.11\end{array}$ & $8.42 \pm 1.57$ & $\begin{array}{l}0.82 \pm \\
1.97\end{array}$ & 0.68 & $\begin{array}{l}9.91 \pm \\
1.26\end{array}$ & $8.11 \pm 0.98$ & $\begin{array}{l}-1.79 \pm \\
1.29\end{array}$ & 0.18 & 0.26 \\
\hline $\begin{array}{l}\text { Galactose } \\
\text { (gr) }\end{array}$ & $\begin{array}{l}0.82 \pm \\
0.25\end{array}$ & $0.88 \pm 0.22$ & $\begin{array}{l}0.06 \pm \\
0.27\end{array}$ & 0.80 & $\begin{array}{l}0.44 \pm \\
0.08\end{array}$ & $0.64 \pm 0.19$ & $\begin{array}{l}0.19 \pm \\
0.20\end{array}$ & 0.36 & 0.71 \\
\hline Lactose (gr) & $\begin{array}{l}5.98 \pm \\
1.13\end{array}$ & $5.00 \pm 1.27$ & $\begin{array}{l}-0.97 \pm \\
1.43\end{array}$ & 0.50 & $\begin{array}{l}3.99 \pm \\
0.96\end{array}$ & $5.71 \pm 1.19$ & $\begin{array}{l}1.72 \pm \\
0.91\end{array}$ & 0.07 & 0.11 \\
\hline Sucrose (gr) & $\begin{array}{l}10.72 \pm \\
2.29\end{array}$ & $\begin{array}{l}12.03 \pm \\
2.27\end{array}$ & $\begin{array}{l}1.30 \pm \\
1.51\end{array}$ & 0.39 & $\begin{array}{l}12.99 \pm \\
2.73\end{array}$ & $\begin{array}{l}11.19 \pm \\
2.17\end{array}$ & $\begin{array}{l}-1.80 \pm \\
1.41\end{array}$ & 0.21 & 0.14 \\
\hline Maltose (gr) & $\begin{array}{l}1.23 \pm \\
0.31\end{array}$ & $1.18 \pm 0.30$ & $\begin{array}{l}-0.04 \pm \\
0.36\end{array}$ & 0.89 & $\begin{array}{l}1.58 \pm \\
0.35\end{array}$ & $1.25 \pm 0.27$ & $\begin{array}{l}-0.33 \pm \\
0.31\end{array}$ & 0.30 & 0.55 \\
\hline Sugar (gr) & $\begin{array}{l}97.30 \pm \\
36.34\end{array}$ & $\begin{array}{l}47.69 \pm \\
5.21\end{array}$ & $\begin{array}{l}-49.60 \pm \\
34.22\end{array}$ & 0.16 & $\begin{array}{l}54.31 \pm \\
5.14\end{array}$ & $\begin{array}{l}48.16 \pm \\
4.93\end{array}$ & $\begin{array}{l}-6.15 \pm \\
3.37\end{array}$ & 0.08 & 0.20 \\
\hline Protein (gr) & $\begin{array}{l}62.24 \pm \\
4.86\end{array}$ & $\begin{array}{l}68.07 \pm \\
5.32\end{array}$ & $\begin{array}{l}5.82 \pm \\
4.77\end{array}$ & 0.23 & $\begin{array}{l}67.40 \pm \\
5.15\end{array}$ & $\begin{array}{l}71.00 \pm \\
5.55\end{array}$ & $\begin{array}{l}3.85 \pm \\
2.73\end{array}$ & 0.17 & 0.72 \\
\hline Fat (gr) & $\begin{array}{l}61.76 \pm \\
6.91\end{array}$ & $\begin{array}{l}64.85 \pm \\
6.44\end{array}$ & $\begin{array}{l}3.08 \pm \\
4.29\end{array}$ & 0.48 & $\begin{array}{l}65.39 \pm \\
3.89\end{array}$ & $\begin{array}{l}69.24 \pm \\
3.26\end{array}$ & $\begin{array}{l}3.59 \pm \\
5.83\end{array}$ & 0.51 & 0.94 \\
\hline $\begin{array}{l}\text { Cholesterol } \\
\text { (mg) }\end{array}$ & $\begin{array}{l}218.41 \\
\pm 35.98\end{array}$ & $\begin{array}{l}238.50 \pm \\
24.29\end{array}$ & $\begin{array}{l}20.09 \pm \\
39.92\end{array}$ & 0.62 & $\begin{array}{l}199.77 \\
\pm 22.35\end{array}$ & $\begin{array}{l}231.15 \pm \\
22.66\end{array}$ & $\begin{array}{l}31.38 \pm \\
32.24\end{array}$ & 0.34 & 0.82 \\
\hline $\begin{array}{l}\text { Saturated } \\
\text { Fat (gr) }\end{array}$ & $\begin{array}{l}14.04 \pm \\
2.18\end{array}$ & $\begin{array}{l}15.04 \pm \\
1.88\end{array}$ & $\begin{array}{l}1.00 \pm \\
1.56\end{array}$ & 0.52 & $\begin{array}{l}13.83 \pm \\
1.34\end{array}$ & $\begin{array}{l}16.99 \pm \\
1.32\end{array}$ & $\begin{array}{l}3.16 \pm \\
1.24\end{array}$ & 0.01 & 0.28 \\
\hline $\begin{array}{l}\text { Mono Fat } \\
(\mathrm{gr})\end{array}$ & $\begin{array}{l}14.73 \pm \\
1.80\end{array}$ & $\begin{array}{l}17.75 \pm \\
2.09\end{array}$ & $\begin{array}{l}3.01 \pm \\
1.61\end{array}$ & 0.07 & $\begin{array}{l}18.48 \pm \\
1.96\end{array}$ & $\begin{array}{l}20.41 \pm \\
1.81\end{array}$ & $\begin{array}{l}1.93 \pm \\
1.83\end{array}$ & 0.30 & 0.66 \\
\hline Poly Fat (gr) & $\begin{array}{l}18.95 \pm \\
2.73\end{array}$ & $\begin{array}{l}61.65 \pm \\
39.84\end{array}$ & $\begin{array}{l}42.70 \pm \\
40.49\end{array}$ & 0.30 & $\begin{array}{l}23.66 \pm \\
2.30\end{array}$ & $\begin{array}{l}24.93 \pm \\
2.19\end{array}$ & $\begin{array}{l}1.26 \pm \\
2.66\end{array}$ & 0.63 & 0.30 \\
\hline $\begin{array}{l}\text { Dietary } \\
\text { Fibers (gr) }\end{array}$ & $\begin{array}{l}17.77 \pm \\
3.75\end{array}$ & $\begin{array}{l}13.58 \pm \\
1.73\end{array}$ & $\begin{array}{l}-4.19 \pm \\
2.23\end{array}$ & 0.07 & $\begin{array}{l}15.15 \pm \\
0.89\end{array}$ & $\begin{array}{l}14.93 \pm \\
0.91\end{array}$ & $\begin{array}{l}-0.22 \pm \\
1.08\end{array}$ & 0.84 & 0.11 \\
\hline $\begin{array}{l}\text { Soluble Fiber } \\
\text { (gr) }\end{array}$ & $\begin{array}{l}0.28 \pm \\
0.04\end{array}$ & $0.33 \pm 0.05$ & $\begin{array}{l}0.04 \pm \\
0.07\end{array}$ & 0.51 & $\begin{array}{l}0.33 \pm \\
0.05\end{array}$ & $0.36 \pm 0.05$ & $\begin{array}{l}0.02 \pm \\
0.06\end{array}$ & 0.66 & 0.84 \\
\hline $\begin{array}{l}\text { Insoluble } \\
\text { Fiber (gr) }\end{array}$ & $\begin{array}{l}1.38 \pm \\
0.26\end{array}$ & $1.59 \pm 0.23$ & $\begin{array}{l}0.21 \pm \\
0.34\end{array}$ & 0.54 & $\begin{array}{l}1.30 \pm \\
0.19\end{array}$ & $1.75 \pm 0.23$ & $\begin{array}{l}0.45 \pm \\
0.26\end{array}$ & 0.09 & 0.57 \\
\hline $\begin{array}{l}\text { Crude Fiber } \\
(\mathrm{gr})\end{array}$ & $\begin{array}{l}8.06 \pm \\
2.61\end{array}$ & $5.46 \pm 1.29$ & $\begin{array}{l}-2.59 \pm \\
1.46\end{array}$ & 0.09 & $\begin{array}{l}5.92 \pm \\
0.38\end{array}$ & $5.57 \pm 0.36$ & $\begin{array}{l}-0.35 \pm \\
0.32\end{array}$ & 0.41 & 0.14 \\
\hline $\begin{array}{l}\text { Calcium } \\
\text { (mg) }\end{array}$ & $\begin{array}{l}638.28 \\
\pm 55.51\end{array}$ & $\begin{array}{l}675.70 \pm \\
75.54\end{array}$ & $\begin{array}{l}29.44 \pm \\
63.62\end{array}$ & 0.64 & $\begin{array}{l}638.28 \\
\pm 50.11\end{array}$ & $\begin{array}{l}662.96 \pm \\
46.99\end{array}$ & $\begin{array}{l}24.68 \pm \\
57.35\end{array}$ & 0.67 & 0.95 \\
\hline $\begin{array}{l}\text { Magnesium } \\
\text { (mg) }\end{array}$ & $\begin{array}{l}227 \pm \\
28.14\end{array}$ & $\begin{array}{l}231.85 \pm \\
30.46\end{array}$ & $\begin{array}{l}3.96 \pm \\
13.61\end{array}$ & 0.77 & $\begin{array}{l}231.28 \\
\pm 12.22\end{array}$ & $\begin{array}{l}238.52 \pm \\
14.46\end{array}$ & $\begin{array}{l}7.24 \pm \\
11.42\end{array}$ & 0.53 & 0.85 \\
\hline
\end{tabular}




\begin{tabular}{|c|c|c|c|c|c|c|c|c|c|}
\hline \multirow[t]{2}{*}{ Variable } & \multicolumn{4}{|c|}{ Cinnamon group $(n=21)$} & \multicolumn{4}{|c|}{ Placebo $(n=22)$} & \multirow{2}{*}{$\begin{array}{l}P \\
\text { Value }^{\mathrm{b}}\end{array}$} \\
\hline & Baseline & $\begin{array}{l}\text { After } \\
\text { intervention }\end{array}$ & $\begin{array}{l}\text { Mean } \\
\text { Difference }\end{array}$ & $\begin{array}{l}\text { P- } \\
\text { Value }\end{array}$ & Baseline & $\begin{array}{l}\text { After } \\
\text { intervention }\end{array}$ & $\begin{array}{l}\text { Mean } \\
\text { Difference }\end{array}$ & $\begin{array}{l}\text { P- } \\
\text { Value }^{a}\end{array}$ & \\
\hline $\begin{array}{l}\text { Vitamin D } \\
\text { (Ug) }\end{array}$ & $\begin{array}{l}0.66 \pm \\
0.18\end{array}$ & $0.65 \pm 0.24$ & $\begin{array}{l}-0.01 \pm \\
0.19\end{array}$ & 0.98 & $\begin{array}{l}0.70 \pm \\
0.19\end{array}$ & $0.97 \pm 0.23$ & $\begin{array}{l}0.27 \pm \\
0.15\end{array}$ & 0.09 & 0.26 \\
\hline $\begin{array}{l}\text { Coffeine } \\
\text { (mg) }\end{array}$ & $\begin{array}{l}74.31 \pm \\
10.15\end{array}$ & $\begin{array}{l}79.19 \pm \\
8.27\end{array}$ & $\begin{array}{l}4.87 \pm \\
7.82\end{array}$ & 0.54 & $\begin{array}{l}66.70 \pm \\
9.39\end{array}$ & $\begin{array}{l}79.39 \pm \\
7.69\end{array}$ & $\begin{array}{l}12.68 \pm \\
8.06\end{array}$ & 0.13 & 0.49 \\
\hline $\begin{array}{l}\text { Physical } \\
\text { activity } \\
\text { (MET- } \\
\text { min/week) }\end{array}$ & $\begin{array}{l}565.93 \\
\pm \\
119.64\end{array}$ & $\begin{array}{l}638.22 \pm \\
223.28\end{array}$ & $\begin{array}{l}72.29 \pm \\
169.72\end{array}$ & 0.42 & $\begin{array}{r}616.59 \\
\pm 183.2\end{array}$ & $\begin{array}{l}683.07 \pm \\
211.87\end{array}$ & $\begin{array}{l}66.47 \pm \\
136.40\end{array}$ & 0.61 & 0.63 \\
\hline
\end{tabular}

\subsection{Change of headache daily result and disability}

After two months of intervention, HDR and disability significantly decreased in the cinnamon group in comparison to the placebo group (Table 3). The mean of HDR significantly reduced in both groups, however, the reduction was significantly greater in the intervention group compared with the control group (137.76 \pm 24.46 to $19.26 \pm 7.85$ and $118.36 \pm 20.13$ to $75.11 \pm 14.00$ in the cinnamon group and placebo groups, respectively, $p=0.006)$. The mean of total scores of disability remarkably reduced from $20.09 \pm 3.02$ to $0.95 \pm 0.47$ (pष0.001) in the cinnamon group, while it did not significantly change in the placebo group (22.63 \pm 2.89 to $16.72 \pm 3.20,(p=0.54))$. The difference between the two groups was statistically significant $(p \otimes 0.001)$. Likewise, the same results were found after adjusting for age and sex as shown in Table 3.

Table 3

Change in disability and headache daily in migraine patients after 2 months of intervention

\begin{tabular}{|c|c|c|c|c|c|}
\hline Parameter & & Cinnamon group $(n=21)$ & Placebo $(n=22)$ & $P$ Value ${ }^{\mathrm{b}}$ & $P$ Value $^{\mathrm{c}}$ \\
\hline \multirow[t]{4}{*}{ MIDAS Score (Day) } & Before & $20.09 \pm 3.02$ & $22.63 \pm 2.89$ & & \\
\hline & After & $0.95 \pm 0.47$ & $16.72 \pm 3.20$ & & \\
\hline & $P$ Value $^{\mathrm{a}}$ & 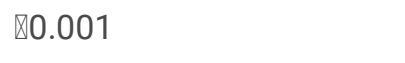 & 0.54 & & \\
\hline & Between-Group Difference & $-20.09 \pm 3.12$ & $-5.90 \pm 2.20$ & $\unrhd 0.001$ & 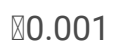 \\
\hline \multirow[t]{4}{*}{ HDR } & Before & $137.76 \pm 24.46$ & $118.36 \pm 20.13$ & & \\
\hline & After & $19.26 \pm 7.85$ & $75.11 \pm 14.00$ & & \\
\hline & $P$ Value $^{\mathrm{a}}$ & $\varangle 0.001$ & 0.006 & & \\
\hline & Between-Group Difference & $118.50 \pm 22.09$ & $43.25 \pm 14.19$ & 0.006 & 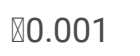 \\
\hline \multicolumn{6}{|c|}{$\begin{array}{l}\text { MIDAS, Migraine Disability Assessment Questionnaire; HDR, Headache Daily Result Note: Data are reported as means } \pm \text { SE } \\
\text { aPaired t-test was used to compare pre-post tests. } \text { Independent samples t-test was used to compare between groups. } \\
\text { cANCOVA test (adjusted for age and sex) }\end{array}$} \\
\hline
\end{tabular}


Table 4

Anthropometric measurement in migraine patients before and after the intervention

\begin{tabular}{|c|c|c|c|c|c|}
\hline Variable & & Cinnamon group $(n=21)$ & Placebo $(n=22)$ & $P$ Value $^{\mathrm{b}}$ & $P$ Value $^{\mathrm{c}}$ \\
\hline \multirow[t]{4}{*}{ Weight (kg) } & Before & $70.02 \pm 2.42$ & $70.50 \pm 3.46$ & & \\
\hline & After & $70.09 \pm 2.57$ & $72.15 \pm 3.54$ & & \\
\hline & $P$ Value $^{\mathrm{a}}$ & 0.60 & $\otimes 0.001$ & & \\
\hline & Between-Group Difference & $-0.17 \pm 0.33$ & $1.64 \pm 0.33$ & $\varangle 0.001$ & 0.001 \\
\hline \multirow[t]{4}{*}{ BMI (kg/m2) } & Before & $26.12 \pm 3.35$ & $26.87 \pm 4.77$ & & \\
\hline & After & $26.06 \pm 3.51$ & $27.50 \pm 4.86$ & & \\
\hline & $P$ Value ${ }^{\mathrm{a}}$ & 0.61 & $\otimes 0.001$ & & \\
\hline & Between-Group Difference & $0.64 \pm 0.57$ & $-0.62 \pm 0.60$ & $\varangle 0.001$ & 0.001 \\
\hline \multirow[t]{4}{*}{ WC (cm) } & Before & $88.79 \pm 1.96$ & $93.00 \pm 2.80$ & & \\
\hline & After & $88.10 \pm 1.96$ & $94.25 \pm 2.86$ & & \\
\hline & $P$ Value ${ }^{a}$ & 0.01 & 0.004 & & \\
\hline & Between-Group Difference & $-1.35 \pm 0.47$ & $1.25 \pm 0.38$ & $\varangle 0.001$ & $\varangle 0.001$ \\
\hline \multirow[t]{4}{*}{$\mathrm{HC}(\mathrm{cm})$} & Before & $105.47 \pm 1.33$ & $101.06 \pm 4.63$ & & \\
\hline & After & $104.92 \pm 1.35$ & $106.07 \pm 1.62$ & & \\
\hline & $P$ Value $^{\mathrm{a}}$ & 0.008 & 0.23 & & \\
\hline & Between-Group Difference & $-0.80 \pm 0.27$ & $5.00 \pm 4.09$ & 0.17 & 0.001 \\
\hline \multirow[t]{4}{*}{ WHR } & Before & $0.84 \pm 0.06$ & $0.88 \pm 0.08$ & & \\
\hline & After & $0.83 \pm 0.06$ & $0.88 \pm 0.08$ & & \\
\hline & $P$ Value $^{\mathrm{a}}$ & 0.10 & 0.33 & & \\
\hline & Between-Group Difference & $-0.006 \pm 0.01$ & $0.003 \pm 0.01$ & 0.06 & 0.008 \\
\hline
\end{tabular}

After intervention with cinnamon, the percentage of participants with Grades III and IV (moderate and severe disability) decreased from 76.18-0\%, whereas those belonging to Grades I and II MIDAS (no or little and mild disability) enhanced from 23.8-95.4\% (Fig. 2). However, in the placebo group, subjects with grade III and IV reduced from $90.90-59.10 \%$ and those belonging to Grades I and II MIDAS increased from $9.00-40.90 \%$, respectively (Fig. 3).

\subsection{Change of anthropometry measurements}

As shown in Table 3 after follow-up, BW and BMI did not change in the intervention group, however, both of them significantly increased in the placebo group. The differences between two groups were statistically significant $(p<0.001)$. Compared with baseline, WC significantly decreased in the cinnamon group and remarkably increased in the control group; the difference between groups was significant ( $p$ 0.001). Nevertheless, after the intervention, although HC significantly decreased in the intervention group, no significant difference was found between groups in the crude model. Likewise, WHR did not significantly change in the intervention compared with control group in the crude model. However, after adjustment for age and sex, ANCOVA test showed that reduction in $\mathrm{HC}$ and WHR in the intervention group was significant compared with control group $(p=0.001$ and $p=0.008$, respectively) (Table 3 ). 


\section{Discussion}

The principal finding of the current study was that cinnamon intake significantly prevented weight gain, increasing BMI, WC, and it reduced $\mathrm{HC}$ and WHR in migraine patients compared with the placebo group. It also significantly reduced the rate of disability in patients by decreasing the HDR in moderate to severe Grades. With regard to the bilateral relationship between obesity and migraine frequency, our findings might be useful in clinical settings to prevent obesity and development of migraine headaches thus improving the functional capacity of migraine patients.

Although the mechanisms contributing to migraine pathophysiology are not completely clear, recent evidence has indicated that the neuroinflammatory state has a critical role in the development of migraine attacks [19]. One of the factors that cause inflammation in the body is the increase in body fat [20]. Also, there has been some evidence that indicates that obesity is comorbid with increasing the severity and frequency of migraine attacks[21].

The weight gain leads to the expansion of adipose tissue and creates a state of chronic low-grade inflammation. Also, the adipose tissue, as a neuroendocrine organ has a role in energy homeostasis and inflammation by adipokines including cytokines, such as tumor necrosis factor TNF-a, interleukins (IL-1, IL-6, IL-10), leptin, resistin, adiponectin, chemokines, such as IL-8, etc. These adipocytokines are one of the suggested causes of the association between obesity and migraine [1]. Most patients suffering from migraines have to use the medications [22]. One side effect of some common drugs used to treat migraines is weight gain which occurs as a result of increased appetite. Therefore, one of the main challenges regarding migraine medication is weight gain and an increase in BMI of patients. $[23,24]$.

Considering several challenges to follow a healthy lifestyle, particularly for a long time for some of the patients, herbal supplements without considerable side effects are an attractive alternative to prevent the side effects of medication such as overweight and obesity [25]. In the current study, we found that cinnamon could be effective to prevent weight gain among the migraine patients, which is in accordance with the results of the previous reviews which showed that cinnamon is an effective agent for weight loss and improving metabolic syndrome $[8,10]$.

To explain these results, it should be mentioned that cinnamon is a potent antioxidant and anti-obesity herb because of its high amounts of polyphenols, flavanols, and cinnamaldehyde [10,26]. Cinnamon lessens glucose absorption in the small intestine by postponing gastric emptying, increasing glucosidase enzymes, and inhibiting ATPase of intestinal brush borders. In addition, activating glycogen synthase and inhibiting glycogen synthase kinase $3 \beta$ lead to decrease glycogenolysis, and increase glycogen synthesis [27]. Also, polyphenolic compounds of cinnamon with anti-obesogenic effects can inhibit lipolysis, lipogenesis, and intestinal lipid absorption [28]. Also, these induce fatty acid oxidation and antagonist at cannabinoid receptors [29]. Therefore, these actions can be effective in decreasing the synthesis and storage of fat and improvement of anthropometric status.

Another compound in cinnamon, which might be effective against increasing weight is Methyl Hydroxy chalcone polymers (MHCP). MHCP causes the enhancement of insulin sensitivity in adipose cells and helps the increasing body metabolism through activating the insulin-receptor kinase and inhibiting the insulin-receptor-phosphatase [8].

The cinnamon consumption can be prevented proinflammatory cytokines production, oxidative stress condition and the risk of developing a number of chronic diseases, including cancer, heart disease, and diabetes caused by insulin resistance in obese migraine patients [10].

In addition to preventing weight gain, in the current study, cinnamon reduced disability in moderate to svere sufferers of migraine by reducing the HDR suggesting cinnamon powder consumption might be a novel approach to reduce the headache disability and control the weight gain in such migraine patients. It seems that cinnamon with anti-inflammatory properties reduces the production of inflammatory cytokines [30] and headache attack time.

Although this study was the first randomized double-blind clinical trial that investigated the effect of cinnamon as a natural, inexpensive, and accessible herbal medicine on anthropometry factors and headache disability in migraine, some limitations should be noted. The duration of the intervention was relatively short and there was no long-term follow-up. Also, the sample size was small. More randomized controlled trails are warranted before causal relationship can be established. Moreover, because of 
ethical issues, the dosage of cinnamon was considered with caution. Therefore, longer and larger trials with different dosages of cinnamon are recommended.

\section{Conclusion}

The results revealed that whole cinnamon consumption was able to control the weight gain and reduced waist and hip circumference in migraine patients. Furthermore, the headache disability and headache daily result significantly decreased in these patients. Our findings implied that cinnamon might be considered as complementary medicine in migraine patients, although further studies are needed to confirm our results.

\section{Abbreviations}

ANCOVA

Analysis of covariance; BMI:Body mass index; BW:Body weight; DASS:Depression Anxiety Stress Scales; HC:Hip circumference; HDR:Headache daily result; ICHD-3:International Classification of Headache Disorders; MIDAS:Migraine Disability Assessment; NAFLD:Non-alcoholic fatty liver disease; NSAIDs:Nonsteroidal anti-inflammatory drugs; SE:Standard error; SPSS:Statistical Package for the Social Sciences; WC:Waist circumference; WHR:Waist-to-hip ratio.

\section{Declarations}

\section{Ethics approval and consent to participate}

The whole protocol of this randomized double-blinded placebo-controlled trial was approved by the Ethics Committee of Isfahan University of Medical Sciences, (ethics code: IR.MUI.RESEARCH.REC.1397.185). The trial has also been registered in the Iranian Center for Clinical Trials (No. IRCT20121216011763N36). Before entering the study, all participants were informed of the study protocol and completed the informed consent.

\section{Consent for publication}

Not applicable.

\section{Availability of data and materials}

The datasets used for this study are available from the corresponding author on reasonable request.

\section{Competing interests}

The authors declared no potential competing interests with respect to the research, authorship, and/or publication of this article.

\section{Funding}

The authors received financial support for the research of this article from the Food Security Research Center of Isfahan University of Medical Sciences grant number 297060, Isfahan, Iran.

\section{Authors' contributions}

$A Z, G A$ and FK designed the study and participated in data acquisition. The data were entered by $A Z$. AH, AZ, MB and MS performed the statistical analyses. MB and AZ drafted the manuscript and GA, FK, MS and AH criticized and reviewed the manuscript. All authors read and approved the final manuscript.

\section{Acknowledgments}

The authors would like to thank the valuable assistance of all participants. They extend their gratitude to the authorities and Food Security Research Center of Isfahan University of Medical Sciences, Isfahan, Iran, for their cooperation. 


\section{Authors' information}

${ }^{1}$ Department of Community Nutrition, School of Nutrition and Food Science, Food Security Research Center, Isfahan University of Medical Sciences, Isfahan, Iran. ${ }^{2}$ Environmental \& Occupational Health, School of Public Health, University of Nevada, Las Vegas, NV, USA. ${ }^{3}$ Department of Neurology, Isfahan University of Medical Sciences, Isfahan, Iran. ${ }^{4}$ Departments of Epidemiology and Biostatistics, School of Health, Isfahan University of Medical Sciences, Isfahan, Iran.

\section{* Corresponding Author:}

Gholamreza Askari MD, PhD, Department of Community Nutrition, School of Nutrition and Food Science, Food Security Research Center, Isfahan University of Medical Sciences, 73461-81746, Isfahan, Iran;

Tel:+983137923171, Fax:+983136688487; Email: askari@mui.ac.ir

\section{References}

1. Di Renzo L, Cammarano A, De Lorenzo A (2018) The missclassification of obesity affects the course of migraine. J Headache Pain 19(1):63. doi:10.1186/s10194-018-0895-6

2. Farhadi Z, Alidoost S, Behzadifar M, Mohammadibakhsh R, Khodadadi N, Sepehrian R, Sohrabi R, Taheri Mirghaed M, Salemi M, Ravaghi H, Behzadifar M (2016) The Prevalence of Migraine in Iran: A Systematic Review and Meta-Analysis. Iranian Red Crescent medical journal 18(10):e40061. doi:10.5812/ircmj.40061

3. Schlesinger S, Neuenschwander M, Schwedhelm C, Hoffmann G, Bechthold A, Boeing H, SchwingshackI L (2019) Food Groups and Risk of Overweight, Obesity, and Weight Gain: A Systematic Review and Dose-Response Meta-Analysis of Prospective Studies. Advances in Nutrition 10(2):205-218. doi:10.1093/advances/nmy092

4. Peterlin BL, Rapoport AM, Kurth T (2010) Migraine and obesity: epidemiology, mechanisms, and implications. Headache 50(4):631-648. doi:10.1111/j.1526-4610.2009.01554.x

5. Miri A, Nasiri M, Zonoori S, Yarahmad F, Dabbagh-Moghadam A, Askari G, Sadeghi O, Asadi M (2018) The association between obesity and migraine in a population of Iranian adults: a case-control study. Diabetes Metab Syndr 12(5):733-736. doi:10.1016/j.dsx.2018.04.020

6. Pavlovic JM, Vieira JR, Lipton RB, Bond DS (2017) Association Between Obesity and Migraine in Women. Curr Pain Headache Rep 21(10):41. doi:10.1007/s11916-017-0634-8

7. Taylor FR (2008) Weight change associated with the use of migraine-preventive medications. Clin Ther 30(6):1069-1080. doi:10.1016/j.clinthera.2008.06.005

8. Mousavi SM, Rahmani J, Kord-Varkaneh H, Sheikhi A, Larijani B, Esmaillzadeh A (2020) Cinnamon supplementation positively affects obesity: A systematic review and dose-response meta-analysis of randomized controlled trials. Clin Nutr 39(1):123-133. doi:10.1016/j.clnu.2019.02.017

9. Rao PV, Gan SH (2014) Cinnamon: a multifaceted medicinal plant. Evid Based Complement Alternat Med 2014:642942. doi:10.1155/2014/642942

10. Mollazadeh $\mathrm{H}$, Hosseinzadeh $\mathrm{H}$ (2016) Cinnamon effects on metabolic syndrome: a review based on its mechanisms. Iran $\mathrm{J}$ Basic Med Sci 19(12):1258-1270. doi:10.22038/ijbms.2016.7906

11. Gupta Jain S, Puri S, Misra A, Gulati S, Mani K (2017) Effect of oral cinnamon intervention on metabolic profile and body composition of Asian Indians with metabolic syndrome: a randomized double -blind control trial. Lipids Health Dis 16(1):113. doi:10.1186/s12944-017-0504-8

12. Bagherniya M, Nobili V, Blesso CN, Sahebkar A (2018) Medicinal plants and bioactive natural compounds in the treatment of non-alcoholic fatty liver disease: A clinical review. Pharmacol Res 130:213-240. doi:10.1016/j.phrs.2017.12.020

13. Billings M, Lopez Mitnik G, Dye BA (2017) Sample size for clinical trials. Oral Dis 23(8):1013-1018. doi:10.1111/odi.12611 
14. Galioto R, O'Leary KC, Gunstad J, Thomas JG, Lipton RB, Pavlovic JM, Roth J, Rathier L, Bond DS (2018) The role of migraine headache severity, associated features and interactions with overweight/obesity in inhibitory control. Int J Neurosci 128(1):63-70. doi:10.1080/00207454.2017.1366474

15. Henry JD, Crawford JR (2005) The short-form version of the Depression Anxiety Stress Scales (DASS-21): construct validity and normative data in a large non-clinical sample. The British journal of clinical psychology 44(Pt 2):227-239. doi:10.1348/014466505x29657

16. Vasudha MS, Manjunath NK, Nagendra HR (2018) Changes in MIDAS, Perceived Stress, Frontalis Muscle Activity and NonSteroidal Anti-Inflammatory Drugs Usage in Patients with Migraine Headache without Aura following Ayurveda and Yoga Compared to Controls: An Open Labeled Non-Randomized Study. Ann Neurosci 25(4):250-260. doi:10.1159/000492269

17. Zandifar A, Asgari F, Haghdoost F, Masjedi SS, Manouchehri N, Banihashemi M, Ghorbani A, Najafi MR, Saadatnia M, Lipton RB (2014) Reliability and validity of the migraine disability assessment scale among migraine and tension type headache in Iranian patients. BioMed research international 2014:978064. doi:10.1155/2014/978064

18. Razeghi Jahromi S, Ghorbani Z, Martelletti P, Lampl C, Togha M (2019) Association of diet and headache. 20 (1):106. doi:10.1186/s10194-019-1057-1

19. Longoni M, Ferrarese C (2006) Inflammation and excitotoxicity: role in migraine pathogenesis. Neurological sciences: official journal of the Italian Neurological Society and of the Italian Society of Clinical Neurophysiology 27 Suppl 2:S107-110. doi:10.1007/s10072-006-0582-2

20. Ford ES, Li C, Pearson WS, Zhao G, Strine TW, Mokdad AH (2008) Body mass index and headaches: findings from a national sample of US adults. Cephalalgia: an international journal of headache 28(12):1270-1276. doi:10.1111/j.14682982.2008.01671.x

21. $10.1155 / 2018 / 6585734$

Huang Q, Liang X (2018) Association between Body Mass Index and Migraine: A Survey of Adult Population in China. 2018:6585734. doi:10.1155/2018/6585734

22. Silberstein SD (2015) Preventive Migraine Treatment. Continuum (Minneapolis, Minn) 21 (4 Headache):973-989. doi:10.1212/con.0000000000000199

23. Young WB (2008) Preventive treatment of migraine: effect on weight. Curr Pain Headache Rep 12(3):201-206. doi:10.1007/s11916-008-0035-0

24. Whyte CA, Tepper SJ (2009) Adverse effects of medications commonly used in the treatment of migraine. Expert Rev Neurother 9(9):1379-1391. doi:10.1586/ern.09.47

25. Wells RE, Baute V, Wahbeh H (2017) Complementary and Integrative Medicine for Neurologic Conditions. Med Clin N Am 101(5):881-893. doi:10.1016/j.mcna.2017.04.006

26. Farhat G, Drummond S, Al-Dujaili EAS (2017) Polyphenols and Their Role in Obesity Management: A Systematic Review of Randomized Clinical Trials. Phytother Res 31(7):1005-1018. doi:10.1002/ptr.5830

27. Hafizur RM, Hameed A, Shukrana M, Raza SA, Chishti S, Kabir N, Siddiqui RA (2015) Cinnamic acid exerts anti-diabetic activity by improving glucose tolerance in vivo and by stimulating insulin secretion in vitro. Phytomedicine: international journal of phytotherapy phytopharmacology 22(2):297-300. doi:10.1016/j.phymed.2015.01.003

28. Mercader J, Palou A, Bonet ML (2011) Resveratrol enhances fatty acid oxidation capacity and reduces resistin and RetinolBinding Protein 4 expression in white adipocytes. J Nutr Biochem 22(9):828-834. doi:10.1016/j.jnutbio.2010.07.007

29. Seely KA, Levi MS, Prather PL (2009) The dietary polyphenols trans-resveratrol and curcumin selectively bind human CB1 cannabinoid receptors with nanomolar affinities and function as antagonists/inverse agonists. J Pharmacol Exp Ther 330(1):31-39. doi:10.1124/jpet.109.151654

30. Ho S-C, Chang K-S, Chang P-W (2013) Inhibition of neuroinflammation by cinnamon and its main components. Food Chem 138(4):2275-2282. doi:10.1016/j.foodchem.2012.12.020

\section{Figures}

Page $12 / 14$ 


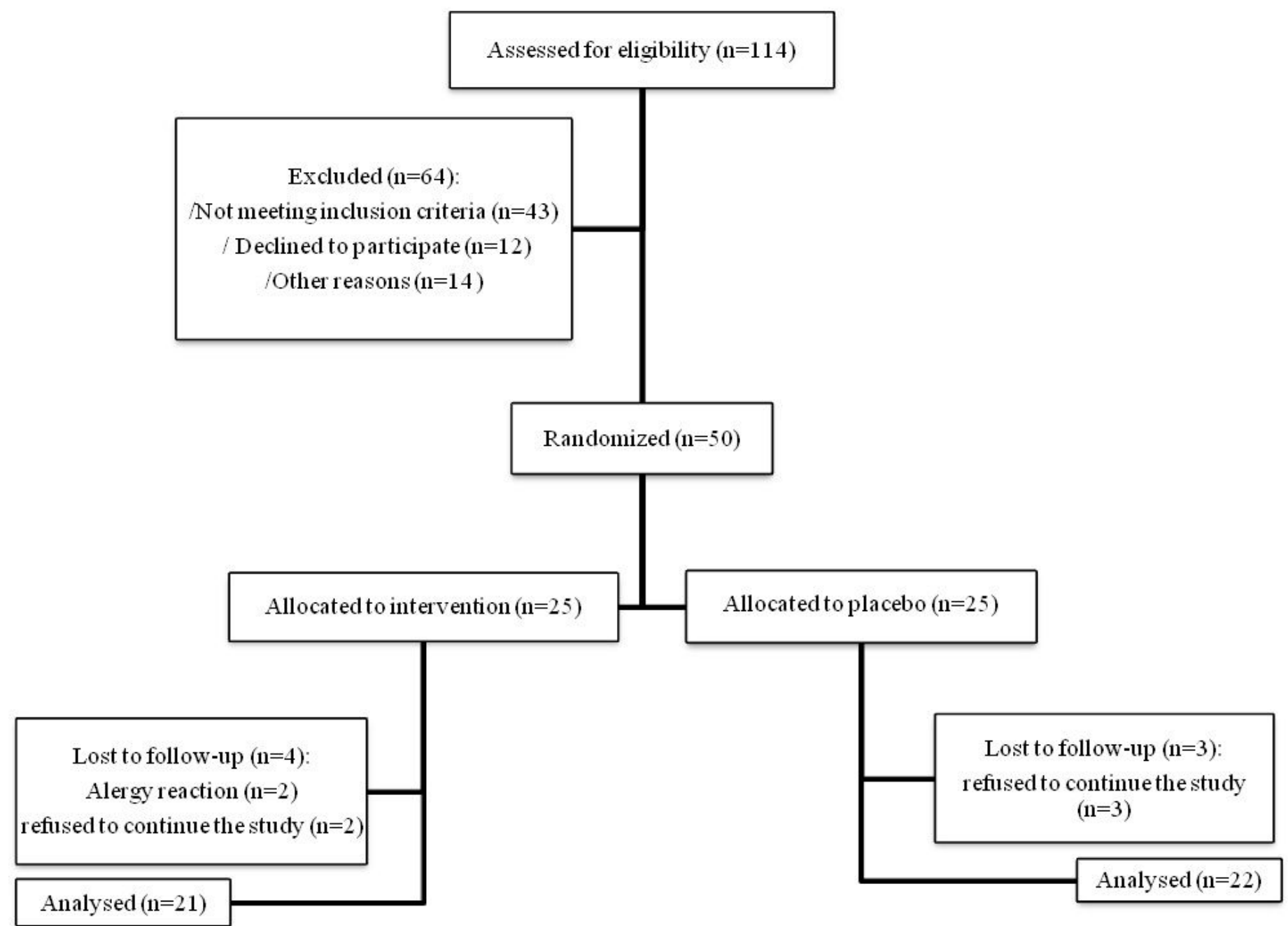

Figure 1

Fig 1. Chart of participants follow-up 


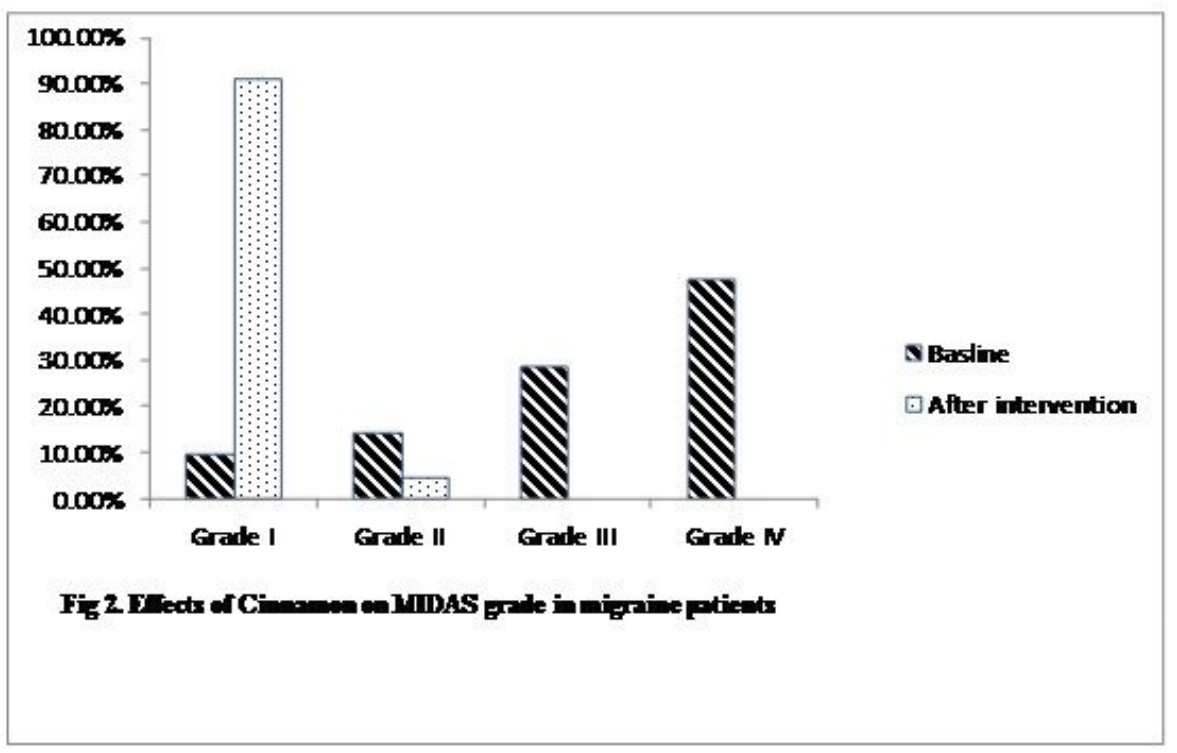

Figure 2

Figure 2

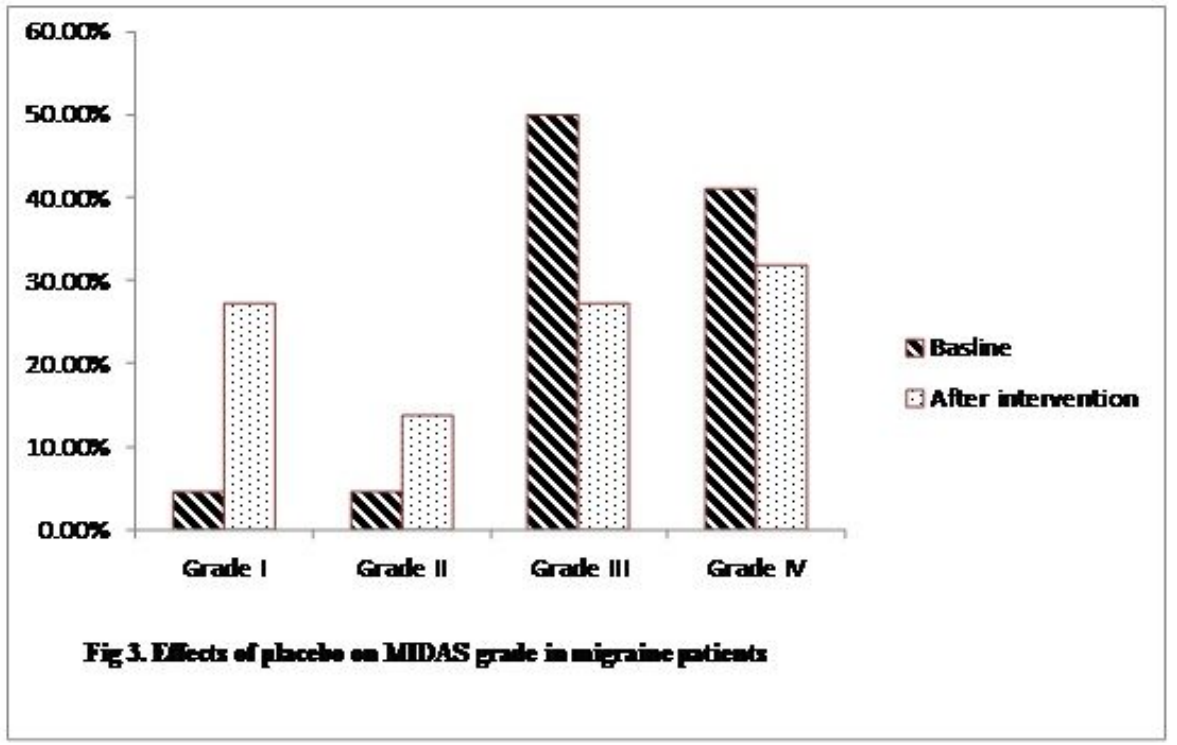

Figure 3

Figure 3 